\title{
The effect of location, mobility and socio-demographic factors on task and time allocation of households
}

\author{
Dick Ettema · Tim Schwanen · Harry Timmermans
}

Published online: 8 July 2006

(C) Springer Science+Business Media B.V. 2006

\begin{abstract}
This paper investigates the role of location factors in task and time allocation at the household level. It is hypothesized that, if time constraints are less binding as a result of living in an urban area or owning more cars, spouses engage more often and longer in out-of-home activities and schedule their activities more independently. The hypotheses are tested with logistic and Cox regression models of activity participation and time allocation on a data set collected in the AmsterdamUtrecht region in the Netherlands. Results suggest that the hypotheses are supported with respect to specific household activity scheduling decisions.
\end{abstract}

Keywords Activity scheduling - Time allocation · Household interactions · Land use factors $\cdot$ Time use $\cdot$ Within-household interactions $\cdot$ Netherlands

\section{Introduction}

In recent years interpersonal linkages in activity participation and time use have attracted increased interest in activity-based travel demand analysis. Focusing on male and female partners within households, two main forms of interaction are commonly distinguished: joint activity engagement and the allocation of householdrelated activities to individual household members. A number of factors have been identified that influence interpersonal, within-household interactions, including socio-demographic variables such as gender, employment status, presence of children, and car availability and work characteristics such as work duration and commute mode (Gliebe and Koppelman 2002; Zhang et al. 2005). To the best of our

D. Ettema $(\varangle) \cdot$ T. Schwanen

Faculty of Geographical Sciences, Urban and Regional Research Centre Utrecht, Utrecht University, PO Box 80.115, Utrecht 3508 TC, The Netherlands

e-mail: d.ettema@geo.uu.nl

H. Timmermans

Urban Planning Group/EIRASS, Eindhoven University of Technology, PO Box 513, Vertigo Building, Eindhoven 5600 MB, The Netherlands 
knowledge, however, no study yet has investigated the role of location factors in within-household interactions in time use and activity participation.

Location factors may be relevant because different environments may offer different opportunities or imply different constraints for time allocation within households. Since studies of the role of spatial context have to date been confined to the individual level (Levinson 1999; Schwanen 2004), this paper examines to what extent within-household interactions in time use depend on households' residential context. More specifically, we investigate how interpersonal associations in the choice and duration of different types of in-home and out-of-home activities vary between residents of higher and lower-density neighborhoods in the AmsterdamUtrecht region, the Netherlands. Because interpersonal linkages are related to individual activity scheduling considerations, we also pay attention to intrapersonal associations in time allocation.

The paper is organized as follows. The next section starts with a brief theoretical exploration of interpersonal linkages in activity duration and formulates hypotheses guiding the empirical analysis. Section "Data" proceeds with a description of the data. The empirical analysis is presented in sections "Activity participation choice" and "Time allocation". Section "Testing the hypotheses" discusses the hypotheses formulated in the context of the results. The paper ends with a set of conclusions and avenues for future research.

\section{Theory and hypotheses}

People's time use is inextricably linked with activity generation and scheduling decisions (Ettema et al. 1995). Knowledge of factors driving activity participation is thus necessary to understand time allocation mechanisms. Although motivations and preferences are important factors driving activity engagement and time use, they are also governed by various interrelated sets of constraints (Arentze and Timmermans 2000; Hägerstrand 1970):

- Biological and physical capability constraints-e.g., the need for sleep and eating;

- Institutional or authority constraints-e.g., facility opening hours;

- Intrapersonal coupling constraints - some activities require individuals to be at a certain location for a certain time period, restricting the time available for other activities;

- Interpersonal coupling constraints-some activities require the presence of other persons, and need to be coordinated with others;

- Monetary budget constraints;

- Characteristics of the space-time environment; and

- Availability of transportation modes, in particular private cars.

Given a set of longer-term choices with respect to residential location, labor participation and vehicle ownership, individuals and households can employ several activity participation strategies to cope with the above constraints. Landau et al. (1981) and Lu and Pas (1999) found that, as the severity of constraints increases, persons will continue to engage in specific activity types (maintenance), and forego participation in activities like leisure, which are less relevant to survival and physical well-being. In addition, when the supply of leisure activities is richer (e.g. in urban areas), more time is spent on leisure activities (Herz 1982; Levinson 1999). In short, 
as constraints become more extensive, individuals have to make more tradeoffs regarding engagement in and the duration of activities.

Tradeoffs made to cope with constraints can, however, exceed the individual level and pertain to the household activity pattern. To understand such tradeoffs, it is useful to make a distinction between independent (personal) activities, shared (joint) activities, and allocated activities, which are household-related activities carried out by one of the partners (Zhang et al. 2005). Engagement in shared and allocated activities depends on the severity of constraints a household faces. Gliebe and Koppelman (2002) suggest that, in situations of more extensive constraints, mandatory activities are more frequently allocated to specific household members-for instance, men concentrating on work and females on household tasks.

A central tenet of this paper is that households in low-density settings and those with fewer than two cars exhibit clearer allocation mechanisms than urbanites and households with two or more cars. Urban environments offer enhanced opportunities for efficient trip and activity chains, allowing women to combine their maintenance tasks with subsistence and leisure activities. This may also stimulate males' independent participation in maintenance activities or the joint engagement of both partners in such activities. However, the impact of residing in a low-density setting can be offset by car accessibility. If a second car is available to the households, both partners are less dependent on the surroundings of the dwelling, and the impact of location may be reduced substantially.

Gliebe and Koppelman (2002) also find that in households with high levels of car accessibility men and women schedule their activities more independently and participate less in shared activities. We believe that the impact of residing in a highdensity environment mirrors that of good car access. This impact may be particularly relevant to leisure activities, because the spatial distribution of leisure facilities (cinemas, restaurants, etc.) is more uneven than that of grocery shops and other daily services.

Given the above theoretical considerations, two lines of reasoning can be pursued with respect to the effects of density and car ownership on household activity scheduling. First, it can be argued that living in an urban environment or owning multiple cars relaxes constraints onto out-of-home activities, leading to:

1.a. More frequent participation in out-of-home activities in general;

1.b. More independence between spouses, implying that activity participation and duration of one spouse is less dependent on the activities of the other spouse;

1.c. More independence between spouses, implying fewer joint activities;

1.d. Fewer intra-personal tradeoffs between activities of one person.

However, the fact that constraints are relaxed in urban settings and when households have access to multiple cars may also lead to a less strict allocation of tasks between spouses, implying that:

2.a. Males are more likely to engage in maintenance activities;

2.b. (Consequently) males trade off work and maintenance activities more often;

2.c. Females engage more frequently in work, leisure and personal business activities;

2.d. (Consequently) females will make more tradeoffs between work, personal business and leisure activities; and 
2.e. Males and females will coordinate work and maintenance tasks, implying that more time spent on these activities by one partner implies less time by the other.

Some of these hypotheses contradict each other, especially hypotheses 1.c. and 2.e. and hypotheses 1.d. and 2.b/2.d. The main question is the extent to which improved accessibility to activities and services due to a higher-density location or the ownership of multiple cars leads to additional activities, replacing the old constraints with new tradeoffs in the context of a busier activity schedule.

The empirical analysis aimed at testing the hypotheses consists of two parts. First, we concentrate on activity engagement through logistic regression models, including density, car ownership, presence of children, activity participation factors and their interactions as explanatory variables. Second, we analyze time allocation conditional on activity participation. Cox regression models are used to this end, again incorporating a wide range of predictor variables. It is recognized that the regression models applied in this study are not capable of representing the full interdependency of activity scheduling in the context of location differences, as some endogenous variables are treated as exogenous ones in the analyses. This paper should therefore be considered as providing initial tests of the potential impact of location factors on task and time allocation.

\section{Data}

The empirical analyses were carried out with the AMADEUS activity/travel diaries that were collected in the Amsterdam-Utrecht region in 2000. This area includes various municipalities, with differing levels of population density, and accessibility to shops and services. In this data collection, 2033 households filled out a 2-day travel/ activity diary. A selection was made to meet the specific requirements of this study. Because we were interested in interactions between spouses, the diaries of singles, and alternative household types (e.g., student houses) were left out. In addition, as we wanted to control for differences in labor market participation, we have selected couples with at least one partner involved in working activities during the questionnaire day, in order to obtain a sample that is comparable with respect to the activities to be allocated. After screening, 707 diary-days of couples are available for the empirical analysis.

Of the couples in our sample $46.4 \%$ have children living with them; $84.6 \%$ own zero or one car and $15.4 \%$ own two or more cars. For each household, the density of the residential neighborhood was determined based on the zipcode of the home location. Besides density, other accessibility indicators were tested in the modeling procedure, such as accessibility to jobs or inhabitants using contour measures. However, density appeared to give the best results in predicting activity participation and duration, possibly because the immediate vicinity of a residence is an important factor for activity participation. Also, the correlations between density and accessibility of jobs $(r=0.64)$ and population $(r=0.72)$ are rather high, suggesting that a higher density implies a better accessibility to jobs and other people. Density data on a four-digit zip code level is available from the Dutch LISA database. The four-digit zip code zones in the study area vary in size between 0.47 and $22.12 \mathrm{~km}^{2}$. Density in the study area ranges between 90 and $19,520 \mathrm{inh} . / \mathrm{km}^{2}$, with a mean of $3650 \mathrm{inh} . / \mathrm{km}^{2}$ 
and standard deviation of $3380 \mathrm{inh} . / \mathrm{km}^{2}$, which suggests a considerable variation in densities. The following variables have been constructed:

$$
\begin{gathered}
\text { HDENSE } \begin{cases}= & 0, \text { if density }<=6000 \mathrm{inh} . / \mathrm{km}^{2} \\
= & 1, \text { if density }>\quad 6000 \mathrm{inh} . / \mathrm{km}^{2}\end{cases} \\
\operatorname{HCAR} \begin{cases}= & 0, \text { if the number of cars in the household is } 0 \text { or } 1 \\
= & 1, \text { if the number of cars in the household is } 2 \text { or more }\end{cases}
\end{gathered}
$$

Our analyses, in which both density and car ownership are allowed to interact with other variables, require the definition of HDENSE and HCAR as binary variables. With respect to density, various cutoff points were tried to find out which density level has the largest impact on time and task allocation. The most significant difference was found between high-density areas ( $>6000$ inhabitants $/ \mathrm{km}^{2}$, in the inner cities of Amsterdam and Utrecht) and other areas. With respect to car ownership, it is argued that the availability of a second car will have a large impact on the accessibility to jobs, stores and other facilities by the other spouse and on household interactions. Fourteen percent of the sample owns two or more cars, while $19.5 \%$ lives in a densely populated area, according to the above definition. Commute time (ComTime), the presence of children younger than 12 (Children) and the respondent's age (in three categories: $\leq 35,36-50$ and $>50$ years) are also included as explanatory variables, because previous studies have suggested their relevance to activity participation and time use (Gliebe and Koppelman 2002; Zhang et al. 2005).

In the diaries, activities were coded in 32 different categories. We have classified these activities into six groups as follows:

1. Work: paid work and voluntary work;

2. Out-of-home household activities (HHout): shopping, serving passengers, other errands;

3. In-home household activities (HHin): childcare, cleaning, repair work;

4. Out-of-home recreation activities (RecOut): sports, recreation, eating out, walking, etc.;

5. In-home leisure (LeisIn): watching TV, reading, having visitors; and

6. Out-of-home personal business (PBout): education, social visits.

\section{Activity participation choice}

In this section, we investigate whether intra- and interpersonal linkages in activity choice are affected by residential location, car ownership and other space-time factors. Logistic regression models are presented with participation in a given activity type as the dependent variable and involvement in other activities by both spouses as explanatory variables (in addition to density, car ownership, commute time, the presence of children and age). To test the effect of location characteristics, interactions between activity participation on one hand and density and car ownership on the other hand are included in the models. All main and interaction effects were included in the model (though only significant effects are included in Table 1). The results are discussed separately for males and females. 
Table 1 Estimated non-standardized coefficients for the logistic regression models of activity participation

\begin{tabular}{|c|c|c|c|c|c|c|c|c|c|c|c|}
\hline & \multicolumn{6}{|l|}{ Male } & \multicolumn{5}{|c|}{ Women } \\
\hline & Work & Hhout & Hhin & Recout & LeisIn & PBout & Work & Hhout & Recout & LeisIn & PBout \\
\hline \multicolumn{12}{|l|}{ Work-M } \\
\hline Work-F & & & & -0.774 & & & & & & & \\
\hline Hhout-M & & & & 0.939 & & & & & & & \\
\hline Hhout-F & 1.054 & & & & & & & & & & \\
\hline $\begin{array}{l}\text { Hhin-M } \\
\text { Hhin-F }\end{array}$ & & & & -2.859 & & & & & & -2.865 & \\
\hline $\begin{array}{l}\text { RecOut-M } \\
\text { RecOut-F }\end{array}$ & & 0.957 & & & & & & & & & \\
\hline LeisIn-M & & & & & & & & & & 4.676 & \\
\hline LeisIn-F & & & -4.973 & & 4.608 & & & & & & \\
\hline PBout-M & & & & & & & & & & & \\
\hline PBout-F & & & & & & & & & & & \\
\hline ComTime-M & & & & & & -0.005 & & & & & \\
\hline ComTime-F & -0.016 & & & & & & 0.054 & -0.047 & & -0.034 & -0.024 \\
\hline Children & & & & -0.595 & & 0.497 & & & -0.566 & 0.761 & 1.808 \\
\hline $\begin{array}{l}\text { High density } \\
2+\text { cars }\end{array}$ & 1.156 & & & & & & 0.547 & & & & \\
\hline Age $\leq 35$ & 1.307 & & -2.447 & & & 0.733 & & & & & \\
\hline age $36-50$ & 1.036 & & & & & & & & & & \\
\hline D-Hhout-M & & & & -1.013 & & & & & & & \\
\hline D-RecOut-M & & -1.028 & & & & & & & & & \\
\hline D-RecOut-F & & & & 1.247 & & & & & & -1.858 & \\
\hline C-work-M & & & & & & -1.432 & 1.432 & & & & \\
\hline C-HHout-M & & & & & & & & -2.205 & & & 1.519 \\
\hline C-HHout-F & & -1.790 & & & & & & & & & \\
\hline C-RecOut-F & & & & & & 1.829 & & & & & -1.662 \\
\hline C-LeisIn-F & & & & & & -2.769 & & 1.859 & & & \\
\hline C-PBout-M & -1.407 & -1.452 & & & & & & & 1.790 & -3.360 & \\
\hline C-PBout-F & & & & & & & & & -1.504 & & \\
\hline $\mathrm{N}$ observations & 707 & 707 & 707 & 707 & 707 & 707 & 707 & 707 & 707 & 707 & 707 \\
\hline Nagelkerke $\rho^{2}$ & 0.19 & 0.10 & 0.54 & 0.15 & 0.52 & 0.17 & 0.23 & 0.24 & 0.16 & 0.43 & 0.31 \\
\hline
\end{tabular}

"-M" = activity by male; "-F" = activity by female; "D-" = interaction with high density; "C-" = interaction with 2+ car ownership

Only coefficients significant at $\alpha=0.05$ are displayed

\section{Males}

The estimation results in Table 1 suggest that men aged 35 or below and those aged 36-50 are more likely to engage in work activities on weekdays than older men, as one would expect. Also, in higher-density areas men are more likely to engage in work activities, possibly because of a better availability of jobs in those areas. The probability of a man working is also affected by the female's activity pattern. If the female has a longer commute time, the male is less likely to work, possibly reflecting a tendency of a more equitable division of household tasks if the female spends more time on work and commuting. However, female engagement in work correlates positively with male work participation, suggesting that males and females coordinate their activity schedules so that working and non-working days are synchronized.

Density is not found to affect interpersonal relationships with respect to male work participation but car ownership does. In households with two or more cars 
male engagement in personal business decreases the probability of working. A possible explanation is that in multiple-car households, both partners engage in work and personal business, leading to the observed tradeoff, whereas task allocation in households with fewer than two cars is more specialized.

For out-of-home household tasks, we find that participation correlates positively with engagement in out-of-home recreation, suggesting that males tend to schedule their activities such that these activity types are combined on the same day. However, this relationship does not hold in high-density settings, suggesting more independence in the scheduling of out-of-home household and recreation activities. The estimation results further suggest that male out-of-home household tasks are less likely to coincide with female out-of-home tasks with increasing car ownership levels. This suggests that car availability reduces the need to strictly allocate out-ofhome tasks to one person, so that either the male or the female performs these tasks. This finding also suggests a reduced need for joint participation if both partners are mobile. With increasing car ownership, the male is less likely to combine out-ofhome tasks with personal business, reflecting a tendency of more independence in the scheduling of non-work activities.

With respect to in-home households tasks, we find that young males are less likely to engage in in-home household tasks. We also find that female participation in leisure decreases the probability of the male pursuing in-home tasks, probably reflecting a tendency of joint engagement in leisure. Neither density nor car ownership affects the male's engagement in in-home household tasks.

With regard to out-of-home recreation, the estimation results suggest that men with children are less likely to engage in recreation, as one would expect. If the female works on a particular day, the male is less likely to engage in recreation, probably because recreation is preferably a joint activity. Consistent with results discussed above, engagement in out-of-home tasks increases the probability of engaging in recreation. Again, we find that this relationship does not hold in highdensity settings, suggesting more independence in the scheduling of non-work activities. If the male pursues in-home household tasks, he is less likely to engage in recreation, implying a tradeoff between these activities. Finally, we find that in highdensity settings, male recreation coincides with female recreation. This probably reflects a preference for joint participation in recreation, which can be realized more easily in high-density settings.

For in-home leisure, only one significant relationship could be detected: male inhome leisure is positively correlated with female in-home leisure, suggesting a preference for joint engagement in in-home leisure. With respect to out-of-home personal business, the estimation results indicate that men with children and men younger than 35 are more likely to engage in personal business. It is also found that the probability of engagement in personal business decreases if commute time is longer, which reflects scheduling constraints. Regarding space-time constraints, car ownership appears to influence the participation in personal business. In households with multiple cars, males are less likely to combine work and personal business, possibly because personal business can be pursued by either household head, reducing the need for the male to combine it with work. Further, we find that female engagement in recreation coincides with male engagement in personal business in multiple-car households. This may reflect more independence in the allocation of tasks among household heads. However, female engagement in in-home leisure 
reduces the probability of male engagement in personal business, again pointing at a preference for joint engagement in in-home leisure.

\section{Females}

The estimation results suggest that the probability of female engagement in work is higher in high-density settings, which may reflect a higher level of job availability in such areas. It is noted that this relationship may also reflect a residential selfselection process, implying that households highly valuing female labor force participation are more likely to reside in neighborhoods offering better opportunities for combining work and household tasks.

The probability of conducting work increases with commute time. This reflects the tendency that commute times for full-time jobs are longer than those of parttime jobs, which can be observed in the data. With respect to car ownership, we find that in households with multiple cars female work participation coincides with male work participation, reflecting a tendency to synchronize work activities between household heads. The same effect was observed for males but not in relation to car ownership, which suggests that ownership of a second car is positively associated with female employment.

The results further suggest that with increasing commute time females are less likely to engage in out-of-home household tasks. In households with multiple cars the probability of female engagement in out-of-home tasks decreases, if the male engages in out-of-home tasks. This reflects a greater flexibility in assigning tasks among household heads in multiple-car households. We also find that in these households engagement in in-home leisure increase the probability of out-of-home tasks.

We have not been able to obtain significant coefficients in models for female in-home task involvement, due to the very large percentage of females $(99.4 \%)$ engaging in this activity. With respect to out-of-home recreation, the estimation results suggest that women with children are less likely to engage in recreation activities, as one would expect. For multiple-car households, we find that male involvement in personal business increases the female's probability of being involved in out-of-home recreation, suggesting that partners schedule their out-of-home activities simultaneously but also more independently. In multiple-car households female engagement in personal business decreases the probability of engagement in recreation. Thus, a higher level of car availability allows women to include both activities in their activity agenda but still requires them to make scheduling tradeoffs between these activity types in the light of restricted time budgets.

With regard to in-home leisure, we find that women with children are more likely to engage in in-home leisure, probably because of lower workforce participation levels and because the coupling constraints associated with the presence of (young) children bind them to the home location for significant parts of the day. The probability of in-home leisure decreases with longer commute times, reflecting time constraints. Also, if the male engages in in-home tasks, the female is less likely to engage in in-home leisure, probably because the latter types of activities are preferably pursued jointly (see the positive correlation between male and female engagement in in-home leisure). We find that the participation in out-of-home recreation reduces the probability of engaging in in-home leisure in higher-density settings. Apparently, residing in a higher-density neighborhood allows women to combine in-home leisure with out-of-home recreation, although tradeoffs between 
these activities have to be made. Finally, we find that the probability of the female spouse in a multiple-car household pursuing in-home leisure is reduced if the male conducts one or more personal business activities. This seems to reflect that the female is less dependent on the availability of a car to go out and does not have to stay at home.

With respect to female personal business, we find that the probability of participation increases with the presence of children. It also decreases with a longer commute time, which appears to reflect the impact of time constraints. In multiplecar households, we find that male engagement in out-of-home task activities increases the probability of female personal business involvement. This may reflect a tendency of partners synchronizing their out-of-home activities, which is possible if multiple cars are available. Consistent with previously discussed results, we also find that in multiple-car households females tend to tradeoff participation against personal business and recreation, suggesting that both activities are included in their activity agendas but are difficult to incorporate together in a single-day activity pattern.

Conclusions regarding the impact of density and car ownership on activity participation choice

The results of the activity participation models are summarized in Fig. 1. Ignoring the effects of density and car availability, we may conclude that males make more tradeoffs between activities than do women. Further, males' activity participation is

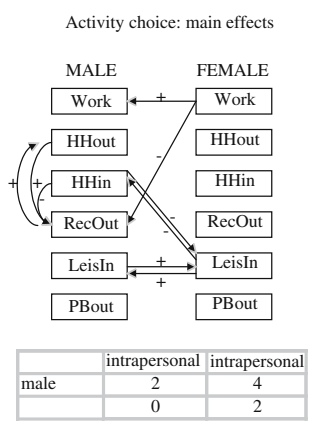

Duration: main effects

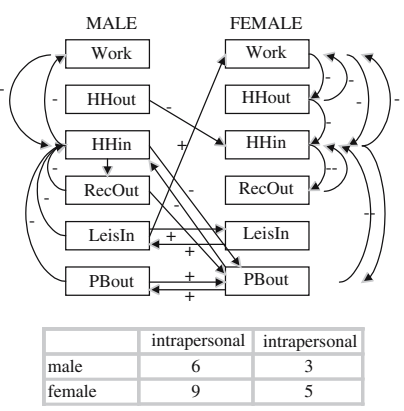

Activity choice: high density

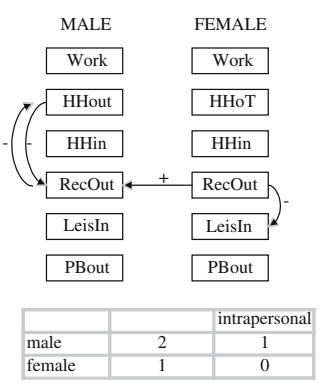

Duration: high density
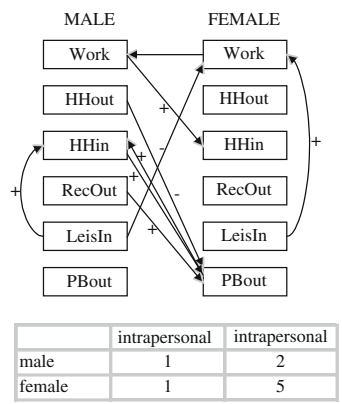

Activity choice: $2+$ cars
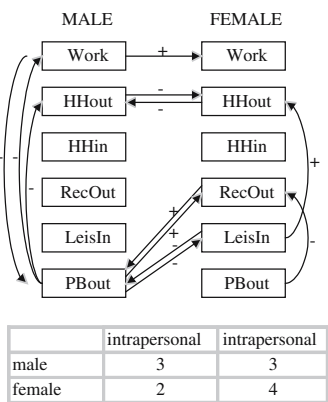

Duration: $2+$ cars

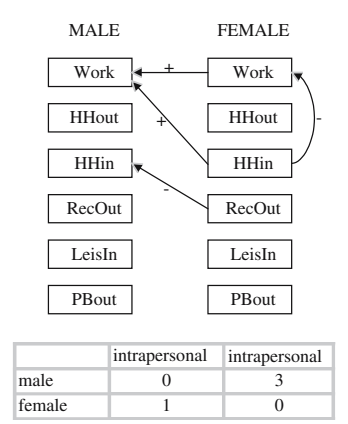

Fig. 1 Effects of density and car ownership on intra- and interpersonal linkages 
more strongly affected by females' activity engagement than the reverse. These findings suggest that males have more choice options with respect to the activities to be included in their schedules. Their choice whether or not to participate in activities depends on tradeoffs between activities and the need to respond to the females' activities. The female on the other hand seems to have a more predetermined activity agenda (due to the need to combine work and maintenance), which is not affected much by the male's activities.

We also find that higher densities primarily affect the male's activity pattern in the sense that more interdependencies between activities occur. The most likely explanation is that more activities become accessible when residing in a higherdensity neighborhood, resulting in additional tradeoffs given space-time constraints

Finally, we find that males in multiple-car households make more tradeoffs than their counterparts in households with fewer cars. These tradeoffs may result from the increase in women's activity participation options due to better car accessibility, requiring the male to engage more frequently in maintenance tasks and personal business. For the female the increased range of options for activity participation leads to more frequent tradeoffs between activities (e.g., recreation and personal business) or induces her to combine specific activities more often (e.g., in-home leisure and out-of-home tasks).

\section{Time allocation}

The effect of location factors on intra- and interpersonal linkages in time allocation is investigated using Cox regression models. Cox regressions are a semi-parametric approach for estimating hazard-based models. The approach is suitable for our purposes because in these models the allocation of time is considered a durationdependent process; the probability to spend an extra time unit on an activity depends on the time already spent on the activity. The Cox regression takes the following general form:

$$
\lambda(t, z)=\lambda_{0}(t) e^{z \beta}
$$

where: $\lambda_{0}$ is an unspecified baseline hazard function; $z$ is a vector of explanatory variables; $\beta$ is a vector of coefficients to be estimated. ${ }^{1}$

In the Cox regressions the time allocated to an activity is the dependent variable, and time allocated to other activities by both spouses serve as explanatory variables, in addition to density, car ownership and other factors. All interactions of time allocation to activities with both density and car ownership are also included in the models (though only significant coefficients are displayed in Table 2).

\section{Males}

The estimation results in Table 2 suggest that younger ( $\leq 35$ years) and middle-aged (36-50 years) men spend more time on work. In addition, if the household has access to two or more cars, males will spend more time on work. If men spend more time on

\footnotetext{
${ }^{1}$ A positive parameter results in a larger value of the hazard function, leading to a shorter activity duration.

篮 Springer
} 


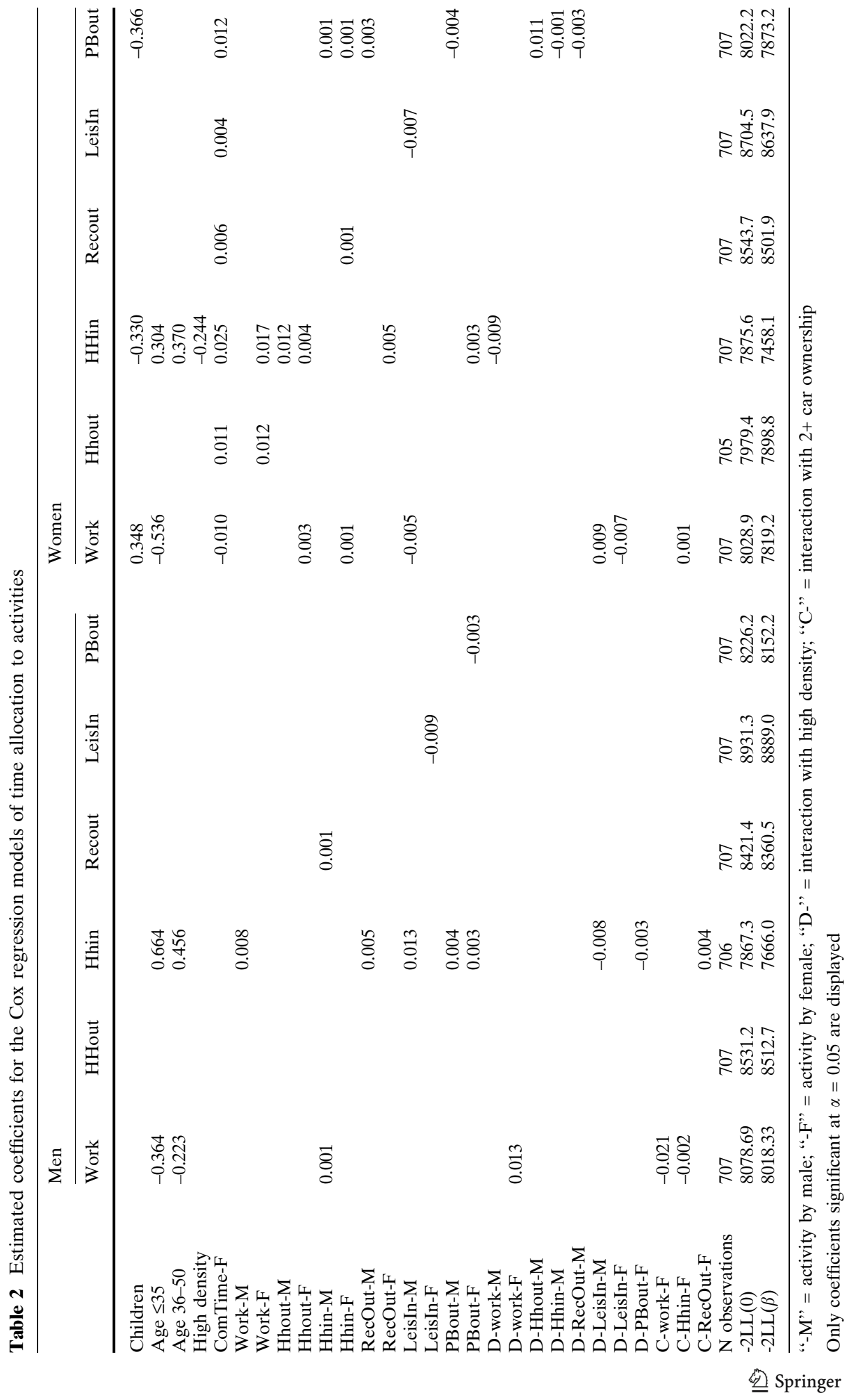


in-home task activities, they tend to spend less time on work, which indicates that tradeoffs are made between these activities. Further, we find that in higher-density settings the male spends less time on work, if the female works longer. This points at a task allocation mechanism at the household level, suggesting that in higher-density settings income-generating activities and other tasks are more evenly distributed between spouses. However, for households with two or more cars we see that the male spends more time on work as the female spouse works less or spends more time on in-home tasks. Collectively, the interactions with density and car ownership suggest that hypothesis 2.e. only holds for households residing in higher-density neighborhoods with one or no car.

No significant effects were found in the model for male out-of-home household tasks. Also, the model as a whole was statistically insignificant. With respect to inhome tasks, we find that men younger than 36 and those aged 36-50 spend less time on in-home tasks than older men, probably because they are more occupied with other activities, such as work. Further, we find that the time allocated to in-home tasks decreases, if males spend more time on work, recreation, in-home leisure and personal business, suggesting that they tradeoff in-home tasks against these activities. Yet, the tradeoff between in-home tasks and in-home leisure is not observed in higher-density settings. Consistent with the results for activity participation, we also find that men spend less time on in-home tasks, as women spend more time on personal business. A possible explanation (supported by the models for personal business) is that men and women tend to pursue personal business activities jointly or at least at the same day. However, this effect cannot be observed in higher-density settings, suggesting a more independent engagement in personal business there. Finally, we find that a male in a multiple-car household spends less time on in-home tasks as his spouse engages longer in recreation. One explanation could be that in such a household car use by the female does not restrict the male's transportation options, so that female out-of-home activities may invoke male out-of-home activities, leading to less time being spent on in-home tasks.

With respect to the male's pursuit of out-of-home recreation, we find that he spends less time on this activity, as he engages more in in-home tasks. This is the same relationship that we found in the in-home task model, which points at a mutual direct tradeoff between these two activities. With respect to in-home leisure, the estimation results reveal that males spend more time on in-home leisure if their spouses do the same. This indicates a preference for jointly engaging in this activity, which is not affected by density or car ownership. We find a positive correlation between time spent on personal business by both partners, which also suggests that they often pursue such activities together.

\section{Females}

With respect to work duration, the estimation results suggest that, as expected, women with children spend less time on work (due to childcare obligations) and that young women ( $\leq 35$ years) spend more time on work. Also, we find that a longer commute time corresponds with longer work duration, as one might expect. Looking at tradeoffs between activities, we find that women spend less time on work as they spend more time on in-home tasks and out-of-home tasks. Thus, the activities competing with work are different for women than for men (in-home tasks and personal business), reflecting general task allocation patterns in households. Yet, 
these tradeoffs depend on space-time conditions. In multiple-car households, women make even more tradeoffs between work and in-home tasks than in households with zero or one car.

Further, we find that the female spouse works longer as the male spends more time in-home leisure. This association does not hold for higher-density settings, pointing at more independence between spouses there. Interestingly, we find that in higher-density settings, more time spent on in-home leisure corresponds with more time being spent on work, suggesting that these activities can be combined well in higher-density settings.

With respect to out-of-home tasks, we see that women spend less time on such activities, if they work or commute longer. The fact that this tradeoff is observed only for females suggests that out-of-home tasks are more women's than men's responsibility (SCP 2004).

Looking at the in-home task model, the results suggest that, as expected, more time is spent on in-home tasks if children are present in the household. Women aged 35 and below and those aged 36-50 spend less time on in-home tasks compared to older women. The estimation results further suggest that the more time the female spends on work, commuting, out-of-home tasks, recreation and personal business, the less she spends on in-home tasks, reflecting tradeoffs that have to be made between these activities. We also find that as the male spends more time on out-ofhome tasks, his partner spends less time on in-home tasks, possibly reflecting situations where the male takes more responsibility of household tasks in general. Interestingly, while women spend more time on in-home tasks as men work more, this effect does not hold for higher-density settings, suggesting more independence between male and female activity schedules in an urban setting.

With respect to out-of-home recreation, we find that women spend less time on recreation with longer commute times or more time spent on in-home tasks, which again reflects time constraints. As for the men, we find that female participation in in-home leisure is positively affected by the time the spouse spends on this activity. Again, this suggests a preference for jointly engaging in this activity. Also, a longer commute time correlates with less time being spent on in-home leisure.

Finally, we find that women spend less time on personal business as they commute longer or participate more in in-home tasks, which reflects tradeoffs given a limited time budget. Interestingly, their time allocation to this activity is affected by males' activity patterns in various ways. Consistent with earlier results, women spend more time on personal business as their partners do the same. In addition, women allocate less time to personal business as their spouse participate longer in recreation or in-home tasks, but these correlations do not hold for higher-density settings, suggesting more independence between spouses in higher-density settings. For couples in higher-density neighborhoods, we also see that women participate for shorter time periods in personal business as males spend more time on out-of-home task activities.

Conclusions regarding the impact of density and car ownership on activity duration

The estimation results of the duration models are summarized in 1 . The results indicate that, in general, women's activity durations are more affected by both intrapersonal linkages (indicating tradeoffs between time spent on various activities) and interpersonal linkages than are men's. A likely explanation is that women in the 
Netherlands are still mainly responsible for maintenance tasks, which have to be combined with subsistence activities (SCP 2004). This results in more tradeoffs between activities. Also, their busy schedules make women more sensitive to males' time allocation than the reverse.

Looking at the role of density, we see that most effects pertain to women's time allocation, in many cases offsetting dependencies on males' time use. Thus, living in a higher-density neighborhood allows women to allocate their time use more independently from their spouses' activity pattern. In contrast, the impact of car ownership is larger for males, making their time allocation to work and in-home tasks more dependent on their spouses' time allocation. This is caused by the second car allowing women to participate more frequently in out-of-home activities. This in turn implies more coordination of the spouses' activity schedules and can restrict males' independent scheduling of activities. For women, the increased opportunities for activity participation due to a second car result in tradeoffs being made between work and in-home tasks.

\section{Testing the hypotheses}

The hypotheses in section "Theory and hypotheses" are displayed in Table 3, together with the empirical findings in support of each hypothesis. This table makes clear that various hypotheses are supported by the estimation results. First, abundant support has been found that individuals engage more often in and allocate more time to work, personal business and out-of-home tasks in situations of fewer constraints, that is, because households reside in a higher-density neighborhood or own multiple cars (H.1.a.). Remarkably, this finding holds for both males and females. Overall, effects of car ownership on out-of-home activities are observed more frequently than for density, although the latter does have a statistically significant impact on male and female work participation. A related finding is that intrapersonal tradeoffs occur less frequently when space-time constraints are less binding (H.1.d).

The findings further suggest that with fewer constraints activity choice and duration are more independent between spouses (H.1.b.). In most cases, this implies that women's activity schedule is more independent from males' activities in higherdensity settings. In addition, the opportunities for joint (recreation) or simultaneous activities (recreation/personal business) increase with higher densities or car ownership levels (H.1.c.).

With respect to task allocation patterns within households, the study supports the hypothesis that fewer constraints lead to a more even distribution of household tasks between spouses. Although males engage more in maintenance activities (H.2.a.), the effect is largest for women, who participate more in activities like work, personal business and recreation (H.2.c.). For both males and females, this implies more tradeoffs in participation and duration for subsistence, maintenance and recreational activities (H.2.b., H.2.d.). Also, activities like work and maintenance are conducted by both spouses rather than being the domain of one of them, which results in more interpersonal interactions (H.2.e.).

It can therefore be concluded that the release of constraints due to living in a high-density area or owning multiple cars results both in increased participation in out-of-home activities and fewer tradeoffs and in a shift in task allocation patterns which leads to more tradeoffs between particular activities of spouses. 
Table 3 Empirical support for various hypotheses

1. Living in urban environment of owning multiple cars releases scheduling constraints, leading to:

a. More participation in out-of-home activities (work, maintenance, leisure) in general:

In higher-density areas, males and females are more likely to work;

In higher-density areas, female work and in-home leisure is combined more easily;

In multiple-car households, females are less likely to engage in in-home task activities;

In multiple-car households, females are more likely to engage in personal business if the male engages in out-of-home tasks;

In multiple-car households, males spend more time on work;

In multiple-car households, males spend less time on in-home tasks if the female spends more time on recreation;

In multiple-car households, females are more likely to combine in-home leisure with out-ofhome tasks.

b. More independence between spouses, implying that activity participation and duration of one spouse do not depend on the activities of the partner:

In higher-density areas, female working time is independent of male in-home leisure time;

In higher-density areas, female in-home task time is independent of male work time;

In higher-density areas, female personal business is independent of male recreation time;

In higher-density areas, male in-home task time is independent of female personal business.

c. Better opportunities for joint or simultaneous participation in activities:

In higher-density areas, spouses engage more often in joint recreation activities;

In multiple-car households, female recreation coincides with male personal business.

d. Fewer tradeoffs between activities of one person:

In higher-density areas, there is no correlation between males' out-of-home task engagement and out-of-home recreation;

In higher-density areas, males do not tradeoff between in-home task and in-home leisure.

2. The fact that constraints are released in higher-density settings and by owning multiple cars may lead to a less strict allocation of tasks between spouses, implying that:

a. Males are more likely to engage in maintenance activities.

In higher-density areas, the male spends more time on in-home tasks if the female engages in personal business.

b. (Consequently) males will make more tradeoffs between work and maintenance activities:

In multiple-car households, males tradeoff between work and personal business;

In multiple-car households, males are less likely to combine out-of-home tasks and personal business;

In multiple-car households, males are less likely to combine out-of-home tasks and recreation.

c. Females will engage more frequently in work, leisure and personal business activities:

In higher-density areas, females are more likely to work;

In multiple-car households, female work coincides with male work;

In multiple-car households, females are more likely to participate in recreation, if the male engages in personal business;

In multiple-car households, females are less likely to participate in in-home leisure if the male engages in personal business;

In multiple-car households, females are more likely to engage in personal business if the male engages in out-of-home tasks;

d. (Consequently) females will make more tradeoffs between work, personal business and leisure activities:

In multiple-car households, females tradeoff personal business and recreation;

In higher-density areas, females tradeoff between in-home leisure and out-of-home recreation.

e. Males and females will coordinate work and maintenance tasks, implying that more time spent by one partner implies less time spent by the other partner:

In multiple-car households, male engagment in out-of-home tasks reduces the probability of female engagement;

In multiple-car households, female engagement in out-of-home tasks reduces the probability of male engagement;

In multiple-car households, more time spent on in-home tasks implies more time spent by the male on work;

In higher-density areas, more female work time implies less male work time. 


\section{Conclusions and discussion}

In this paper, we have analyzed household activity participation and time allocation as a function of socio-demographics, mobility and especially location factors. Overall, the results indicate the existence of various intra- and interpersonal linkages in activity choice and time allocation. In comparison with other daily scheduling factors, location factors and car ownership do not play a very large role. Where location factors are of importance, they concern specific intra- or interpersonal scheduling decisions. They seem to be more important for women than for men when activity durations are concerned. This may reflect the fact that women tend to be responsible for combining work and housekeeping tasks to a greater degree, implying that they are under larger time pressure and have to make more tradeoffs between activities. On the other hand, the effect of location factors is largest for men as far as activity participation is concerned. This may reflect that their activity agendas are less well defined and depend on interpersonal task allocation.

The analyses provided support for the hypothesis that increased local accessibility in higher-density areas facilitates involvement in out-of-home activities, which holds for both men and women. In addition, a higher density is positively correlated with more independent participation of spouses in out-of-home recreation and in-home household tasks, as well as with joint or simultaneous engagement in out-of-home recreation and out-of-home personal business. However, living in higher-density areas and having more cars available can also contribute to a more even distribution of subsistence and maintenance tasks among spouses, implying that they have to make more tradeoffs among various activities and that their activity patterns are more interdependent. Thus, the release of constraints seems to lead not only to increased options for activity participation but also to busier schedules implying more tradeoffs and interdependencies.

\section{References}

Arentze, T.A., Timmermans, H.J.P.: ALBATROSS: A Learning-Based Transportation Oriented Simulation System. Eindhoven, EIRASS (2000)

Ettema, D.F., Borgers, A.W.J., Timmermans, H.J.P.: Competing risk hazard model of activity choice, timing, sequencing, and duration. Transport. Res. Rec. 1493, 101-109 (1995)

Gliebe, J.P., Koppelman, F.S.: A model of joint activity participation between household members. Transportation 30, 49-72 (2002)

Hägerstrand, T.: What about people in regional science? Region. Sci. Assoc.Papers 24, 7-21 (1970)

Herz, R.: The influence of environmental factors on daily behaviour. Environ. Plan. A 14, 1175-1193 (1982)

Landau, U., Prasher, J.N., Hirsh, M.: The effect of temporal constraints on household travel behavior. Environ. Plan. A 13, 435-448 (1981)

Levinson, D.M.: Space, money, life-stage, and the allocation of time. Transportation 26, 141-171 (1999)

Lu, X., Pas, E.I.: Socio-demographics, activity participation and travel behavior. Transport. Res. A 33, 1-18 (1999)

Schwanen, T.: The determinants of shopping duration on workdays in the Netherlands. J. Transport Geogr. 12, 35-48 (2004)

SCP (Social and Cultural Planning Office) (2004) Trends in Time. The Use and Organisation of Time in the Netherlands, 1975 - 2000. The Hague: SCP. Available at: http://www.scp.nl/english/ publications/books/9037701965.shtml.

Zhang, J., Timmermans, H., Borgers, A.: A model of household task allocation and time use. Transport. Res. B 39, 81-95 (2005)

铂 Springer 


\section{Authors biography}

Dick Ettema is assistant professor in the Department of Human Geography and Planning at Utrecht University in The Netherlands. His research interests center around the analysis and modelling of activity patterns, including the impact of land use on activity scheduling, household interactions and longer term adaptation processes.

Tim Schwanen is assistant professor in urban geography at Utrecht University in the Netherlands. He currently works on the project 'Coping with Coupling Constraints' about how dual-career households combine work and domestic responsibilities and manage uncertainty about the timing of everyday activities. This research is financed by the Innovational Research Incentives Scheme of the Netherlands Organisation of Scientific Research (NWO).

Harry Timmermans is professor of urban planning. His research interests include modelling of choice behavior in a variety of application contexts and the development of decision support systems. 